\title{
Experimental Study on the Aperture of Geomagnetic Location Arrays
}

\author{
Xiaojun Zhang, Xiyuan Kang, Xin Chen, Zhuoshan Geng, Liming Fan, Quan Zheng, Hua Lv, \\ and Chong Kang $(\mathbb{D}$
}

College of Science, Harbin Engineering University, Harbin 150001, China

Correspondence should be addressed to Chong Kang; kangchongheu@163.com

Received 9 October 2018; Accepted 16 January 2019; Published 7 March 2019

Academic Editor: Valerie Renaudin

Copyright ( 2019 Xiaojun Zhang et al. This is an open access article distributed under the Creative Commons Attribution License, which permits unrestricted use, distribution, and reproduction in any medium, provided the original work is properly cited.

\begin{abstract}
A method of locating a magnetic target based on geomagnetic total field is proposed. In the method, a conjugate gradient algorithm is introduced to eliminate the time-varying and uneven spatial distribution of geomagnetic total field. Then a structure of the measuring array of geomagnetic total field is designed. In the measuring array, the array aperture is a primary factor for the conjugate gradient algorithm. To determine an optimal aperture, we analyze the relationship between the array aperture and the localization accuracy. According to the localization theory based on geomagnetic total field, we simulate the process of determining an optimum array aperture. Based on the simulation, we propose the basis and principle of determining the optimum array aperture. To prove it, we use optically pumped magnetometers with different array apertures to carry out the experiments of locating a car in a suburb. Through the experiment, we get the experimental relationship between apertures and location accuracy. And the relationship agrees with the theory. The result shows that the method is feasible to determine the optimum aperture.
\end{abstract}

\section{Introduction}

It is important to locate targets by magnetic field in geological monitoring, energy and mineral exploration, rescue of plane crash, antisubmarine detection, and medical diagnosis [1-3]. In measuring geomagnetic total field, optically pumped magnetometers have the advantages of high resolution, long detection range, and no temperature drift [4-6]. Therefore, it is feasible to locate the magnetic target by using the array of optically pumped magnetometer. In order to improve the location accuracy, the time-varying and uneven spatial distribution of geomagnetic total field must be eliminated $[7,8]$. In this paper, a double gradient algorithm is introduced to eliminate the distribution. In the method, some magnetic field changes caused by targets have also been filtered out. In particular, when two sensors are close to each other, the magnetic field of targets will be filtered out more. On the one hand, all the information is missing when the array aperture is zero. On the other hand, the disturbance field cannot be filtered when the array aperture is infinite. Therefore, it is necessary to determine an optimal array aperture which filters the interference field to the maximum extent and filters the target field to the minimum extent.

The double gradient algorithm comprehensively describes the actual situation of target's magnetic field gradient, so the algorithm is not affected by the filtering of some signal gradient. The difference is only that the measured value of the decimal point has been moved forward. For the sensors with high precision and high resolution, the accuracy of locating a target will not be affected as long as the mantissa can be measured. However, if the sensor accuracy and resolution are low, the mantissa will not be detected. It means that the dual gradient algorithm cannot detect the remote or small target. An optimum aperture is an equilibrium point of the double gradient algorithm. Base on the optimum aperture, we can filter the interference field to the maximum extent and filter the target field to the minimum extent. It is impossible to eliminate the magnetic field generated by the 
target completely. It is only required that the desired accuracy of position is within an expected range.

The optimum array aperture is related to sensor precision, resolution, noise of instrument and environment, target magnetic moment, detection position, and detection range. These quantities should be estimated and expected before designing the positioning system, so the calculation method of the optimum aperture can be given according to these estimates.

\section{Method of Determining an Array Aperture}

2.1. The Location Algorithm. When the distance between a target and a sensor is more than 2-3 times the scale of the target, the target can be regarded as a magnetic dipole [9-11]. To measure the magnetic field gradient of a target in a $3 \mathrm{D}$ space and locate it, the array can be composed of several general field sensors as shown in Figure 1. The distance $D$ between a sensor and origin $\mathrm{O}$ is defined as the array aperture.

$\mathbf{T}_{0}$ is the magnetic field vector with no target, $T_{0}$ is its magnitude, and the local magnetic dip and magnetic declination are, respectively $\theta$ and $\varphi$, so the unit direction vector of $\mathbf{T}_{0}$ is $\vec{e}=[\cos \theta \cdot \cos \varphi \cos \theta \cdot \sin \varphi \sin \theta]$. For $i=1,2,3 \ldots$, $T_{i}$ is the measurement of the magnetic sensor $i$ at $\left(x_{i}, y_{i}, z_{i}\right)$ with a magnetic target. The target is at $(x, y, z)$. Then, the displacement vector from the target to the sensor $i$ is $\vec{r}_{i}=\left(x_{i}-x\right) \vec{i}+\left(y_{i}-y\right) \vec{j}+\left(z_{i}-z\right) \vec{k}$, and $r_{i}$ is its magni- tude. $\mathbf{P}_{m}$ is the magnetic moment vector of the target. $P_{m}$ is its magnitude. $\alpha$ and $\beta$ denote the offset angle and the tilted angle of $\mathbf{P}_{m}$, respectively. The magnetic field vector $\mathbf{B}_{i}$ denotes the field generated by the target at the $i$ th magnetic sensor. In the far-field condition, $\left|\mathbf{B}_{i}\right|$ is much less than $T_{0}$, so the total field sensor measurement is $T_{i} \approx T_{0}+\vec{e} \cdot \mathbf{B}_{i}[12]$.

According to the magnetic dipole model in far field, the magnetic field $\mathbf{B}_{i}$ generated by the target can be expressed as

$$
\mathbf{B}_{i}=\frac{\mu_{0}}{4 \pi r_{i}^{3}}\left(\frac{3\left(\mathbf{P}_{m} \cdot \vec{r}_{i}\right) \vec{r}_{i}}{r_{i}^{2}}-\mathbf{P}_{m}\right)
$$

Substituting

$$
\begin{aligned}
& P_{m}=P_{m}\left[\begin{array}{c}
\cos \alpha \cos \beta \\
\cos \alpha \sin \beta \\
\sin \alpha
\end{array}\right], \\
& \mathbf{r}_{\mathbf{i}}=\left[\begin{array}{c}
x-x_{i} \\
y-y_{i} \\
z-z_{i}
\end{array}\right]
\end{aligned}
$$

into equation (1), $\mathbf{B}_{i}$ can be expressed as

$$
\mathbf{B}_{i}=\frac{\mu_{0} P_{m}}{4 \pi r_{i}^{3}}\left(\frac{3\left(\left(x-x_{i}\right) \cos \alpha \cos \beta+\left(y-y_{i}\right) \cos \alpha \sin \beta+\left(z-z_{i}\right) \sin \alpha\right)}{r_{i}^{2}}\left[\begin{array}{c}
x-x_{i} \\
y-y_{i} \\
z-z_{i}
\end{array}\right]-\left[\begin{array}{c}
\cos \alpha \cdot \cos \beta \\
\cos \alpha \cdot \sin \beta \\
\sin \alpha
\end{array}\right]\right)
$$

Then, the scalar measurement of the $i$ th magnetic sensor can be expressed as

$$
\begin{aligned}
T_{i}= & T_{0}+\frac{\mu_{0} P_{m}}{4 \pi r_{i}^{3}} \cdot\left(\frac{3\left[\left(x-x_{i}\right) \cos \alpha \cos \beta+\left(y-y_{i}\right) \cos \alpha \sin \beta+\left(z-z_{i}\right) \sin \alpha\right]}{r_{i}}\right. \\
& \times \frac{\left[\left(x-x_{i}\right) \cos \theta \cos \varphi+\left(y-y_{i}\right) \cos \theta \sin \varphi+\left(z-z_{i}\right) \sin \theta\right]}{r_{i}} \\
& -[\cos \theta \cdot \cos \varphi \cos \alpha \cdot \cos \beta+\cos \theta \cdot \sin \varphi \cos \alpha \cdot \sin \beta+\sin \theta \sin \alpha]) .
\end{aligned}
$$

It can be seen that formula (5) is a scalar equation about the variables $x, y, z, P_{m}, \alpha$, and $\beta$. $(x, y, z)$ denotes the spatial position of the target. $P_{m}$ can be used to estimate the size of the target. Due to high nonlinear function, it is difficult to get an analytical solution of the variables. Generally, we can obtain a numerical solution by solving the optimization problem. Therefore, we solve equation (5) fast and accurately using the software LINGO [13]. 


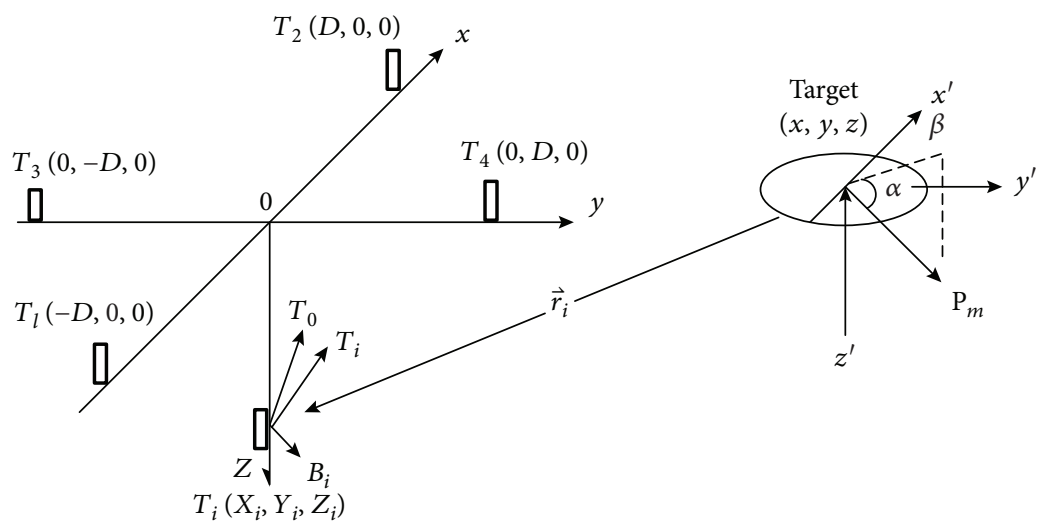

FIGURE 1: Schematic diagram of the location array.

2.2. Double Gradient Algorithm. The time-varying and uneven spatial distribution may have an influence on locating a target. The influence must be eliminated in magnetic field measurement. The measured value of $i$ th sensor located at ( $\left.x_{i}, y_{i}, z_{i}\right)$ is $T_{i}\left(t_{0}, x_{i}, y_{i}, z_{i}\right)$ at time $t_{0}$ and $T_{i}\left(t, x_{i}, y_{i}, z_{i}\right)$ at time $t$. The measurements of $j$ th sensor are expressed in the same way. $\Delta T_{i j}$ is a double gradient of the time and space of geomagnetic measurements. The double gradient $\Delta T_{i j}$ is expressed as

$$
\begin{aligned}
\Delta T_{i j}= & T_{i}\left(t, x_{i}, y_{i}, z_{i}\right)-T_{j}\left(t, x_{j}, y_{j}, z_{j}\right) \\
& -\left[T_{i}\left(t_{0}, x_{i}, y_{i}, z_{i}\right)-T_{j}\left(t_{0}, x_{j}, y_{j}, z_{j}\right)\right] .
\end{aligned}
$$

Through (5) and (6), we can locate the target location by $\Delta T_{i j}$. In this way, the time-varying and uneven spatial distribution can be filtered out [12]. Therefore, we use double gradient $\Delta T_{i j}$ in the whole experiment. The magnetic field generated by the target is also filtered to some extent when using the double gradient. Therefore, the optimum array aperture is necessary to filter the interference field to the maximum extent and the target field to the minimum extent.

2.3. Principle of Determining the Optimum Aperture. In the linear motion of a target, its magnetic moment vector is fixed, which can be regarded as unknown constants. According to formula (6), $\Delta T_{i j}$ is a function of variables $x, y, z, D$. It can be expressed as

$$
\Delta T_{i j}=f(x, y, z, D) .
$$

The uncertainty at $(x, y, z)$ is expressed as

$$
\Delta\left(\Delta T_{i j}\right)=\left.\left(\left|\frac{\partial f}{\partial x}\right| \cdot \Delta x+\left|\frac{\partial f}{\partial y}\right| \cdot \Delta y+\left|\frac{\partial f}{\partial z}\right| \cdot \Delta z\right)\right|_{x, y, z}
$$

In the formula, $\Delta\left(\Delta T_{i j}\right)$ cannot be less than the instrument and ambient noise $\Delta T_{\min }$. It means that there is a lower limit $\Delta T_{\min } . \Delta x, \Delta y, \Delta z$ denote the positioning accuracy of the target in three directions. It is assumed that all the positioning accuracy in three directions is $\Delta r$, then

$\Delta\left(\Delta T_{i j}\right)=\left.\left(\left|\frac{\partial f}{\partial x}\right|+\left|\frac{\partial f}{\partial y}\right|+\left|\frac{\partial f}{\partial z}\right|\right)\right|_{x, y, z} \cdot \Delta r=g(x, y, z, D) \cdot \Delta r \geq \Delta T_{\min }$.

In order to improve the positioning accuracy, the function $g(x, y, z, D)$ should be extremely high. When the target is at a certain point $(x, y, z), g(x, y, z, D)$ is only a function of $D$. Therefore, there is an optimal value of the aperture $D$, which makes $g(x, y, z, D)$ reach a maximum. It means that, when $\partial g(x, y, z, D) / \partial D=0$, the optimal value of the theory is obtained. It can be expressed as

$$
\left|\frac{\partial^{2}\left(\Delta T_{i j}\right)}{\partial x \partial D}\right|+\left|\frac{\partial^{2}\left(\Delta T_{i j}\right)}{\partial y \partial D}\right|+\left|\frac{\partial^{2}\left(\Delta T_{i j}\right)}{\partial z \partial D}\right|=0 .
$$

According to the above principle, the optimal aperture can be determined by the following procedures:

(1) Determinate the instrument and ambient noise $\Delta T_{\min }$

(2) Set the location accuracy $\Delta r$ of the experiment

(3) For specific $(x, y, z)$, solve for a set of $D$ in the function $g(x, y, z, D) \geq \Delta T_{\min } / \Delta r$

(4) Find the intersection of $D$ sets corresponding to all $(x, y, z)$ points in the location space

(5) Choose the minimum value of $D$ in the intersection to minimize the scale of the array

Step 4 can be omitted when a coordinate point or a target in a local area is concerned.

In theory, it is not possible in theory to find the corresponding $D$ value for all $(x, y, z)$ in the location space. Therefore, it can be calculated by dividing grid points in the measurement space, and the spacing of grid points can be set according to the locating requirement, so that the computation workload can be reduced. In practice, we can calculate the optimum aperture of several representative points in the 


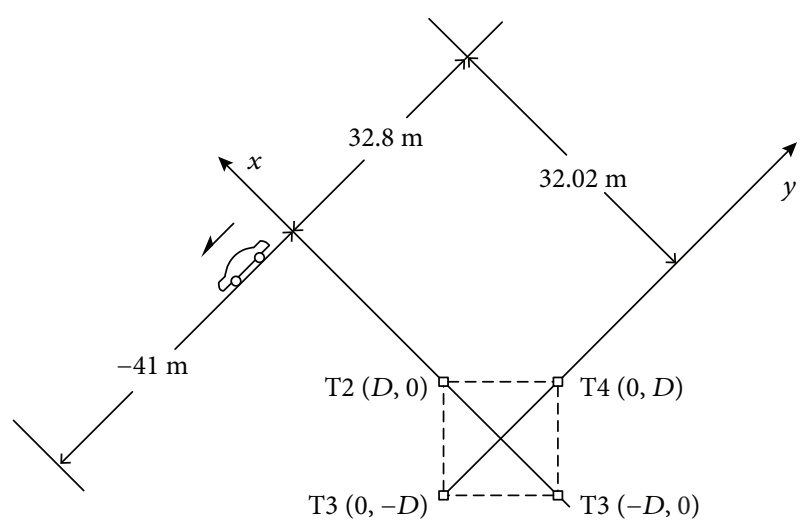

(a)

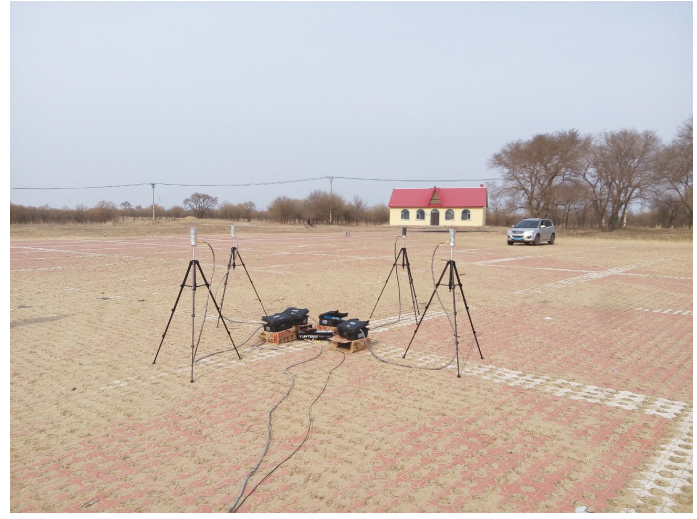

(b)

Figure 2: Schematic diagram and photo of the experimental area.

region, or the three-dimensional space can be reduced to two dimensions or one dimension. Then the computation can be further reduced.

\section{Simulation}

3.1. Simulation of the Experimental Environment. As shown in Figure 2, four optically pumped magnetometers $T_{1}, T_{2}$, $T_{3}, T_{4}$ form a square array.

The aperture of the array is $D$. The target car moves at a constant velocity from $y=32.8 \mathrm{~m}$ to $y=-41 \mathrm{~m}$ along a parallel line $32.02 \mathrm{~m}$ from the $y$ axis. Local magnetic dip is $63.3^{\circ}$, and local magnetic declination is $10.34^{\circ}$.

The curve of the magnetic field changed over time in the experimental area, as shown in Figure 3. The horizontal axis represents time $t$, and the unit is s. The vertical axis is the double gradient value of magnetic field, and the unit is $\mathrm{nT}$. The array aperture is $D=6 \mathrm{~m}$. The red line in Figure 3 is the measurement value T1 of sensor 1 (for the convenience of making the drawing, each value minus $55315 \mathrm{nT})$. T1 includes the geomagnetic field which varies with time and the magnetic field generated by the target car which varies by car's movement. Because the geomagnetic field itself varies greatly over time, the red line cannot be used to judge when a car passed. The blue line is the data of $\Delta T_{14}$, which is processed by the double gradient of formula (6). The time-varying and uneven spatial distribution of the geomagnetic field is filtered using the double gradient of the magnetic field. The sensitivity of sensor CS-L is $0.6 \mathrm{pT} / \mathrm{Hz}^{1 / 2} @ 1 \mathrm{~Hz}$ (rms). The peak value of the instrument noise is $2 \mathrm{pT}$, and the bandwidth is $0.1 \mathrm{~Hz}$. The measurement noise generated by the double gradient of local environmental magnetic field is $10 \mathrm{pT}$. The measurement value of the car magnetic moment is $P_{m}=405 \mathrm{Am}^{2}, \alpha=0.592 \mathrm{rad}$, and $\beta=3.74 \mathrm{rad}$.

\subsection{Results of the Simulation}

3.2.1. Results with the Optically Pumped Magnetometers. Figure 4 shows the curve of the partial derivative $\partial\left(\Delta T_{34}\right) / \partial$ $y$ and $D$ in simulation that $y=-40 \mathrm{~m}$. The horizontal axis

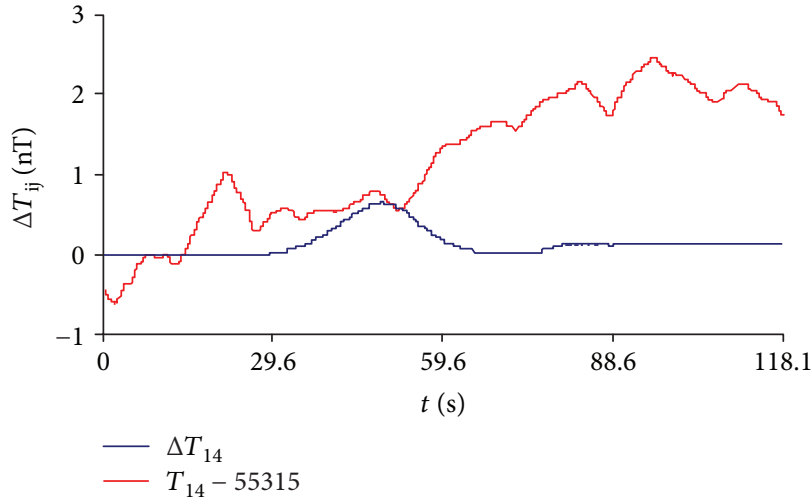

FIgURE 3: Curve of magnetic field variation over time in experimental area.

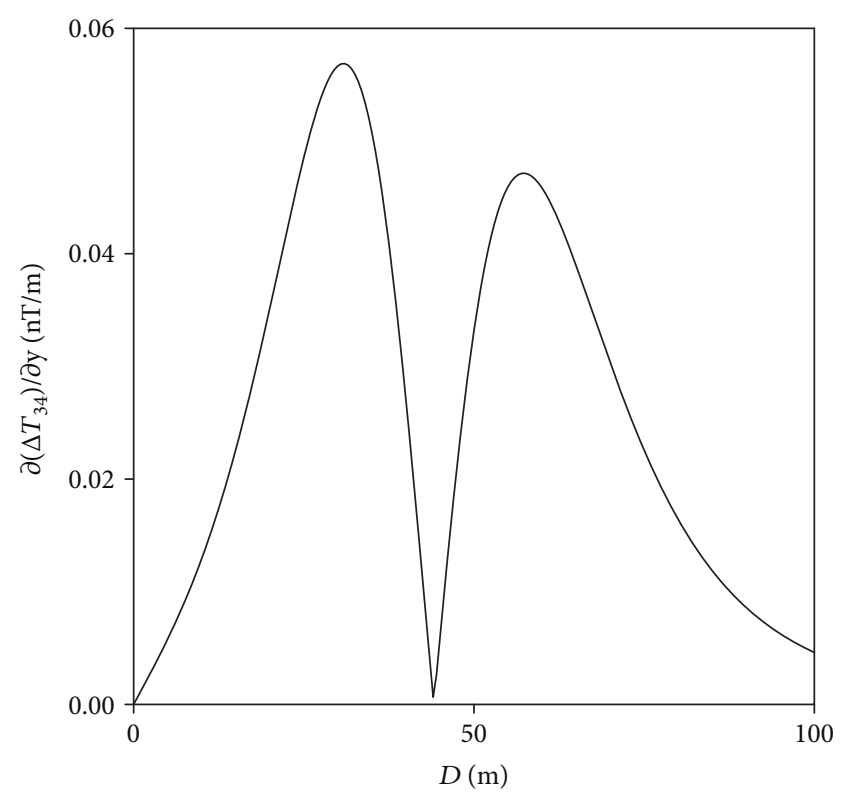

Figure 4: Curves of $\partial\left(\Delta T_{34}\right) / \partial y$ and $D$. 


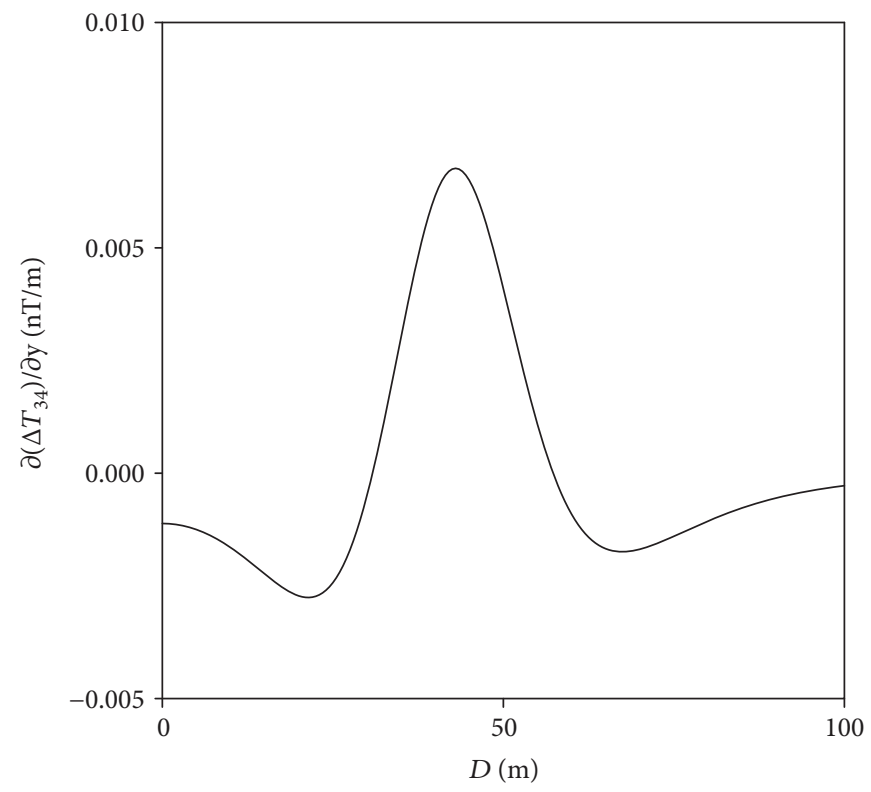

Figure 5: Curves of $\partial^{2}\left(\Delta T_{34}\right) / \partial y \partial D$ and $D$.

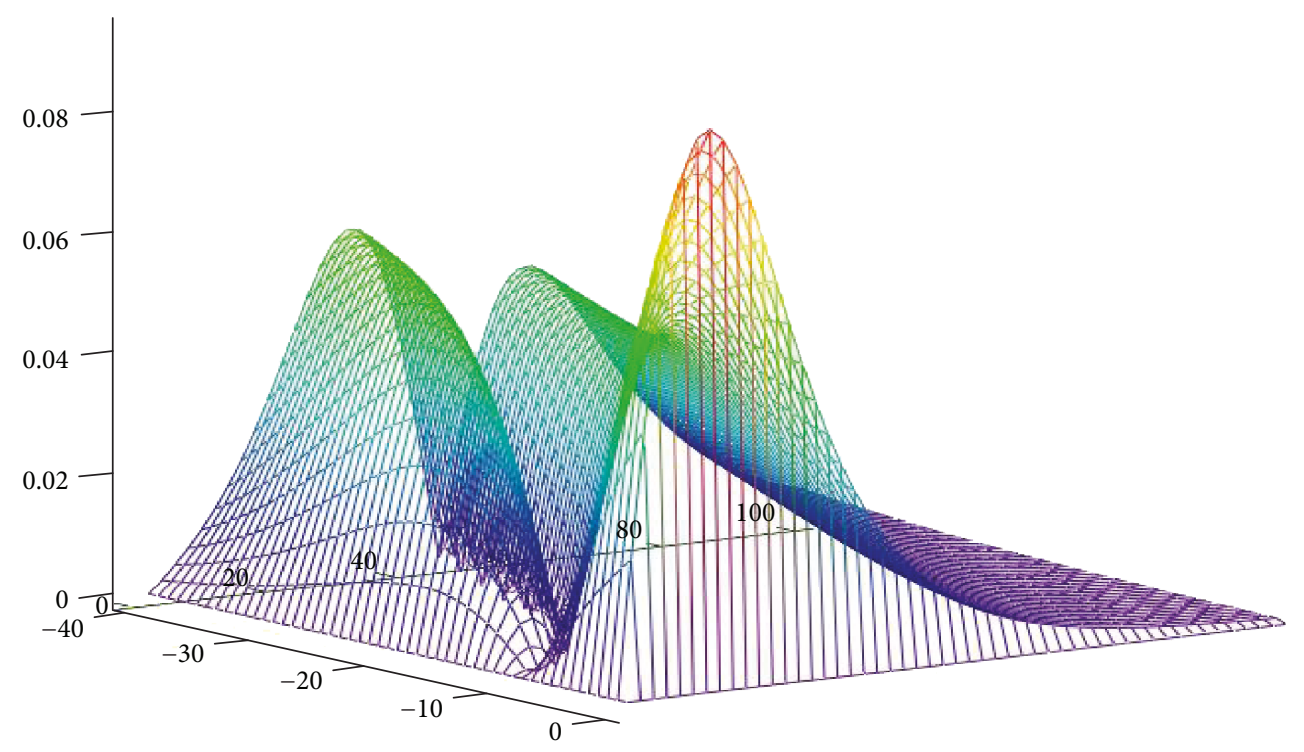

Figure 6: Three-dimensional curves of $\partial\left(\Delta T_{34}\right) / \partial y, y$, and $D$.

is $D$ value, unit $\mathrm{m}$, value range $(0 \mathrm{~m}, 100 \mathrm{~m})$. The vertical axis is the value of $\partial\left(\Delta T_{34}\right) / \partial y$, unit $\mathrm{nT} / \mathrm{m}$, variation range $(0 \mathrm{nT} / \mathrm{m}, 0.1 \mathrm{nT} / \mathrm{m})$. When $D=32 \mathrm{~m}$, the curve has a $\max -$ imum value. Thus, the theoretical optimum aperture $D$ is $32 \mathrm{~m}$, and the location accuracy of the target is the highest. The noise of environment and instrument is $10 \mathrm{pT}$. If the location accuracy is $1 \mathrm{~m}$, it is required that $\partial\left(\Delta T_{34}\right) / \partial y \geq$ $10 \mathrm{pT} / \mathrm{m}$ in the curve of Figure 4. In fact, $D \in(9,43.5) \cup$ $(47,91) \mathrm{m}$ satisfies the location accuracy, so $D_{\min }=9 \mathrm{~m}$ is chosen to minimize the array scale. Figure 5 is the curve of the second mixed partial derivative $\partial^{2}\left(\Delta T_{34}\right) / \partial y \partial D$ and $D$ in simulation that $y=-40 \mathrm{~m}$. The horizontal axis is $D$ value, unit $\mathrm{m}$, value range $(0 \mathrm{~m}, 100 \mathrm{~m})$. The vertical axis is the value of $\partial^{2}\left(\Delta T_{34}\right) / \partial y \partial D$, unit $\mathrm{nT} / \mathrm{m}^{2}$, variation range $\left(0 \mathrm{nT} / \mathrm{m}^{2}, 0.1 \mathrm{nT} / \mathrm{m}^{2}\right)$. There are two $D$ values corresponding to the second derivative $\partial^{2}\left(\Delta T_{34}\right) / \partial y \partial D=0$, $32 \mathrm{~m}$, and $59 \mathrm{~m}$. There is a maximum value for the first derivative $\partial\left(\Delta T_{34}\right) / \partial y$ at $D=32 \mathrm{~m}$.

Figure 6 is a surface graph of $\partial\left(\Delta T_{34}\right) / \partial y, y$, and $D$. The blue horizontal axis in the horizontal plane is $y$ value, unit $\mathrm{m}$, simulation range from $0 \mathrm{~m}$ to $-40 \mathrm{~m}$. The green horizontal axis in the horizontal plane is $D$ value, unit $\mathrm{m}$, simulation range from $0 \mathrm{~m}$ to $100 \mathrm{~m}$. The vertical axis is the value of $\partial\left(\Delta T_{34}\right) / \partial y$, unit $\mathrm{nT} / \mathrm{m}$, variation range from $0 \mathrm{nT} / \mathrm{m}$ to $0.1 \mathrm{nT} / \mathrm{m}$. It can be seen that $\partial\left(\Delta T_{34}\right) / \partial y$ varies with $y$ and $D$. It is a three-dimensional surface graph.

Figure 7 is a graph of $\partial\left(\Delta T_{34}\right) / \partial y$ and $D$ with different $y$ values. The horizontal axis is $D$ value, unit $\mathrm{m}$, value range 


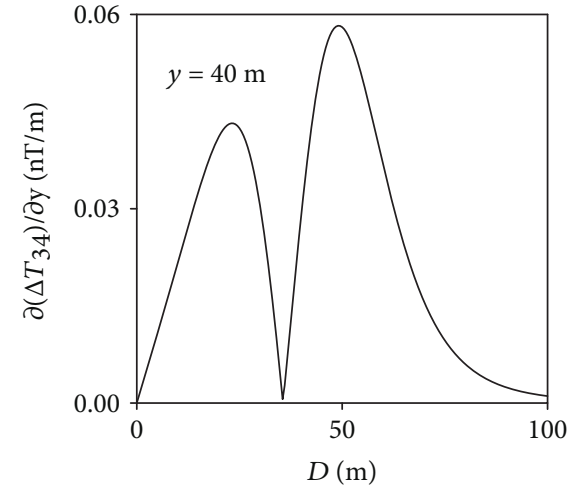

(a) Curves when $y=40$

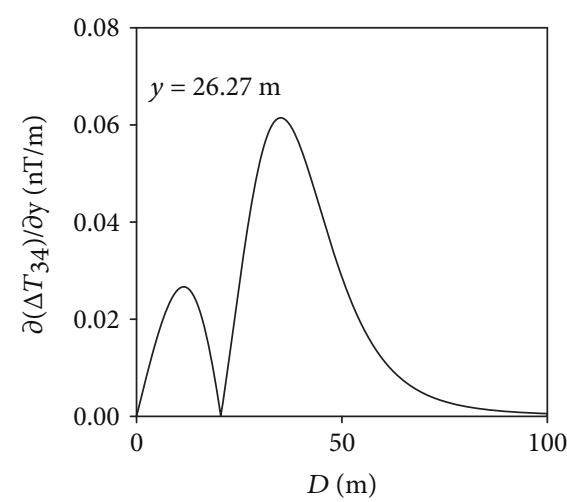

(c) Curves when $y=26.27$

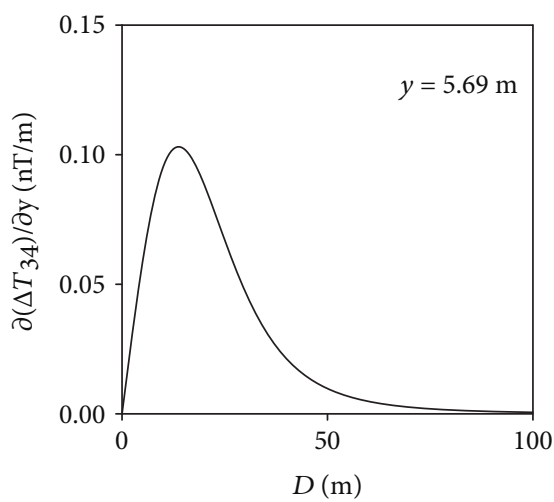

(e) Curves when $y=5.69$

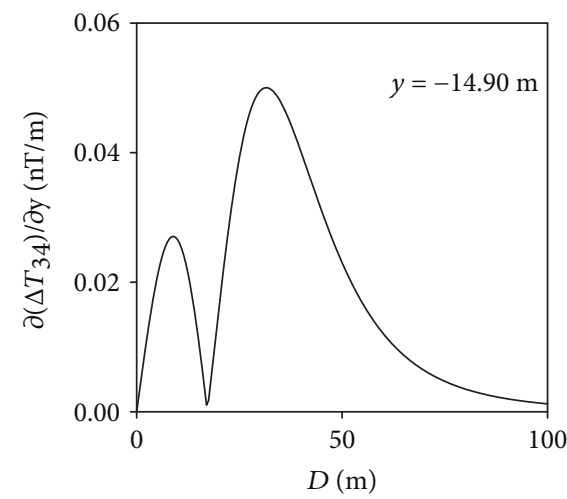

(g) Curves when $y=-14.90$

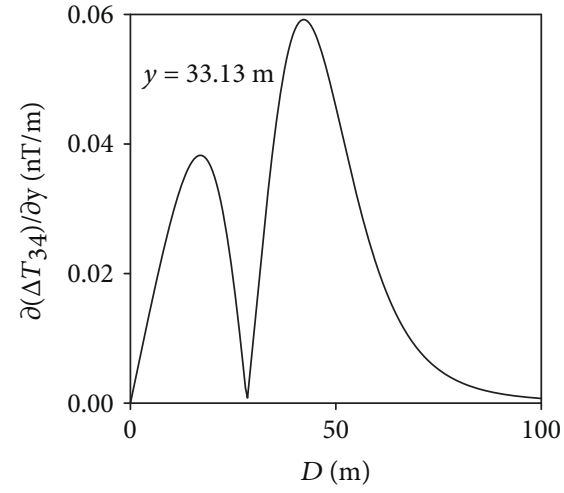

(b) Curves when $y=33.13$

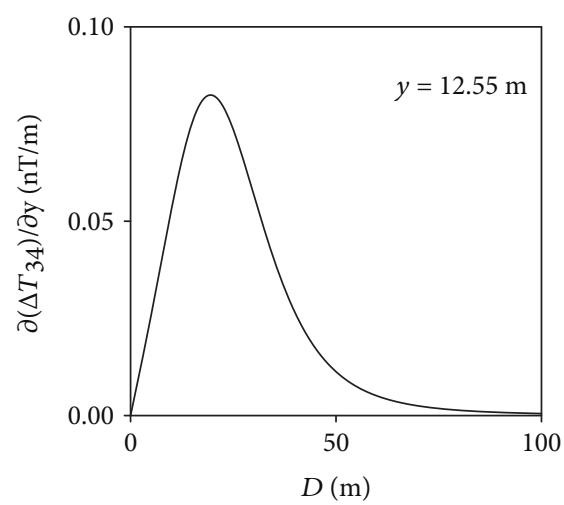

(d) Curves when $y=12.55$

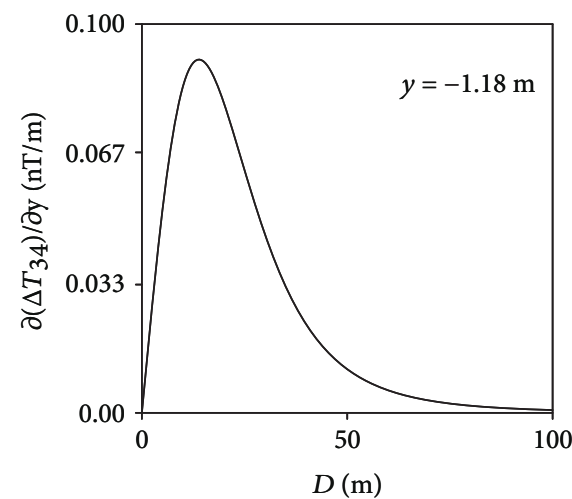

(f) Curves when $y=-1.18$

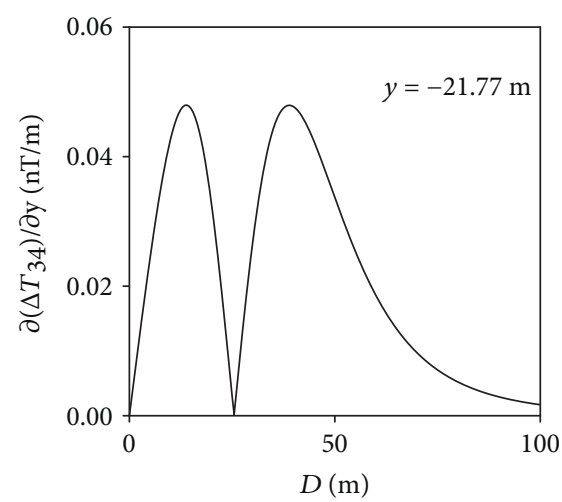

(h) Curves when $y=-21.77$

Figure 7: Continued. 


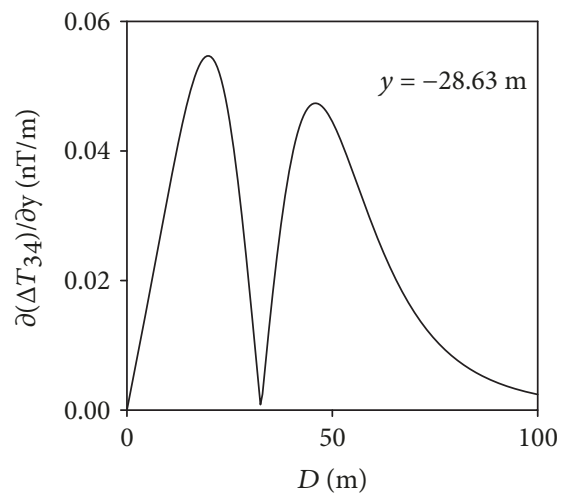

(i) Curves when $y=-28.63$

Figure 7: Curves of $D$ with different $y$ values.

TABle 1: Value of $D$ which meets the precision requirement.

\begin{tabular}{lc}
\hline$y(\mathrm{~m})$ & $D(\mathrm{~m})$ \\
\hline 40.00 & $D \in(4.5,32.5) \cup(36,75.5)$ \\
33.13 & $D \in(3.5,25) \cup(28.5,68.5)$ \\
26.27 & $D \in(3.5,16.5) \cup(21,62.5)$ \\
12.55 & $D \in(2,54)$ \\
5.69 & $D \in(1,53)$ \\
-1.18 & $D \in(1,56)$ \\
-14.90 & $D \in(2.5,16.5) \cup(21,67)$ \\
-21.77 & $D \in(2.5,25) \cup(28.5,73.5)$ \\
-28.63 & $D \in(3.5,32.5) \cup(36,80)$ \\
\hline
\end{tabular}

from $0 \mathrm{~m}$ to $100 \mathrm{~m}$. The vertical axis is the value of $\partial\left(\Delta T_{34}\right)$ $/ \partial y$, unit $\mathrm{nT} / \mathrm{m}$, variation range from $0 \mathrm{nT} / \mathrm{m}$ to $0.1 \mathrm{nT} / \mathrm{m}$. In Figure 7 , the curve varies with $D$ when the target $y$ value is $40.00 \mathrm{~m}, 33.13 \mathrm{~m}, 26.27 \mathrm{~m}, 12.55 \mathrm{~m}, 5.69 \mathrm{~m},-1.18 \mathrm{~m}$, $-14.9 \mathrm{~m},-21.77 \mathrm{~m}$, and $-28.63 \mathrm{~m}$.

Therefore, the maximum $D$ of theory changes with the target location $y$. When the environmental noise is $10 \mathrm{pT}$ and the location accuracy is $1 \mathrm{~m}$, the range of $D$ value which meets the precision requirement is calculated according to the data in Figure 7, as shown in Table 1.

According to the requirements of accuracy, the intersection of $D$ value set in Table 1 is $D \in(4.5,16.5) \cup(21,25) \cup$ $(28.5,32.5) \cup(36,53) \mathrm{m}$. In addition to satisfying the requirement of location accuracy, the array scale should be minimized to make it more flexible, following the principle of Section 2.3. The minimum value $D_{\min }=4.5 \mathrm{~m}$ is selected as the optimal aperture of CS-L array.

3.2.2. Results with Fluxgate Magnetometers. We also performed the simulation with the array of HS-MS-FG3S fluxgate magnetometers made in China. The frequency-domain noise of this magnetometer is $10 \mathrm{pT} / \mathrm{Hz}^{1 / 2} @ 1 \mathrm{~Hz}$ per channel. When we measure the total geomagnetic field by it, the total noise of three channels is $17.32 \mathrm{pT} / \mathrm{Hz}^{1 / 2} @ 1 \mathrm{~Hz}$, and the total noise is $20 \mathrm{pT} / \mathrm{Hz}^{1 / 2} @ 1 \mathrm{~Hz}$ after superimposing with the white noise of geomagnetic field. When the positioning
TABle 2: Value of $D$ which meets the precision requirement.

\begin{tabular}{lc}
\hline$y(\mathrm{~m})$ & $D(\mathrm{~m})$ \\
\hline 40.00 & $D \in(9.5,32) \cup(38.5,67)$ \\
33.13 & $D \in(7,24.5) \cup(31.5,60.5)$ \\
26.27 & $D \in(6.5,16) \cup(24,54)$ \\
12.55 & $D \in(4,43)$ \\
5.69 & $D \in(2,40.5)$ \\
-1.18 & $D \in(2,41.5)$ \\
-14.90 & $D \in(5,12.5) \cup(21,52)$ \\
-21.77 & $D \in(4,22) \cup(29,58.5)$ \\
-28.63 & $D \in(6.5,29.5) \cup(36,65)$ \\
\hline
\end{tabular}

accuracy is $1 \mathrm{~m}$, the range of $D$ value satisfying the accuracy requirement is calculated according to the data in Figure 7, as shown in Table 2.

The intersection of $D$ value set in Table 2 which meets the requirements of accuracy is $D \in(9.5,12.5) \cup(38.5,40.5) \mathrm{m}$. In addition to satisfying the requirement of location accuracy, the array scale should be minimized to make it more flexible, following the principle of Section 2.3. The minimum value $D_{\text {min }}=9.5 \mathrm{~m}$ is selected as the optimal aperture of HS-MS-FG3S array. It can be seen that the increase of instrument noise has an effect on the array aperture, which makes the optimal aperture of the array increase accordingly.

\section{The Location Experiment and Data Analysis}

We set four CS-L cesium optically pumped magnetometers in the suburb as shown in Figure 2. The target moved along the planned trajectory parallel to the $y$ axis direction in the horizontal plane, $x=32.02 \mathrm{~m}$. The horizontal axis in Figure 8 is the values of $y, y \in(-41,32.8) \mathrm{m}$. The vertical axis is the double gradient values of total geomagnetic field, which is with the unit $\mathrm{nT}$, and $t_{0}$ is the moment $y=32.8 \mathrm{~m}$. The experiment is divided into four situations: $D=1 \mathrm{~m}, 2 \mathrm{~m}$, $4 \mathrm{~m}$, and $6 \mathrm{~m}$. Red symbol $\bigcirc$ in Figure 8 represents the $y$ value of double gradient $\Delta T_{14}, \Delta T_{24}, \Delta T_{34}$ measured by experiment. The blue line in Figure 8 is the theoretical curve of 


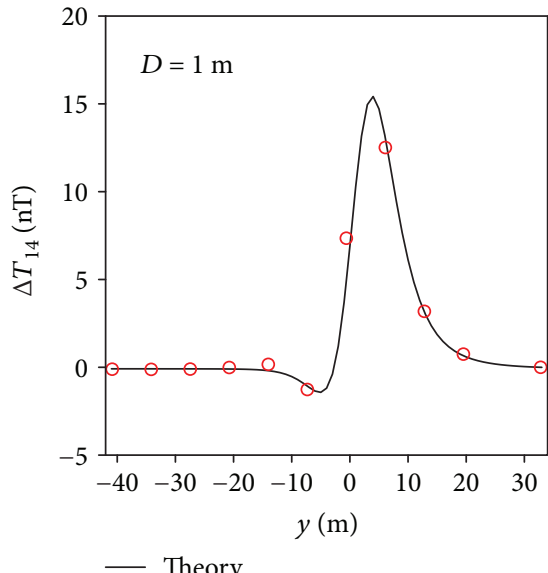

- Theory

- Experiment

(a) $D=1 \mathrm{~m}$, curves of $\Delta T_{14}$ and $y$

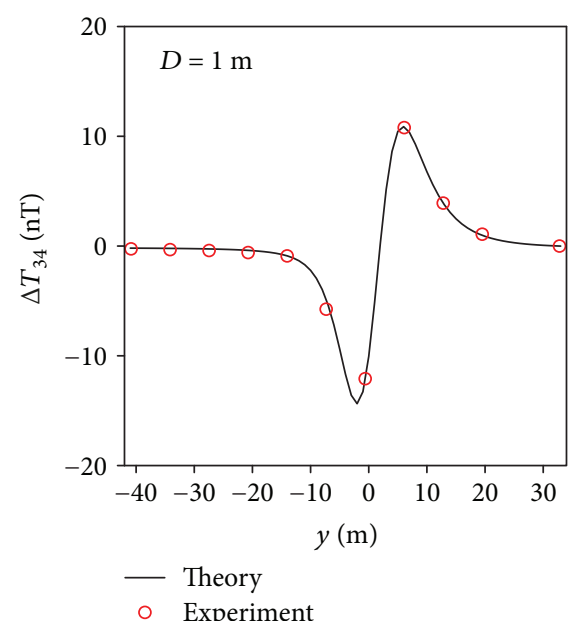

(c) $D=1 \mathrm{~m}$, curves of $\Delta T_{34}$ and $y$

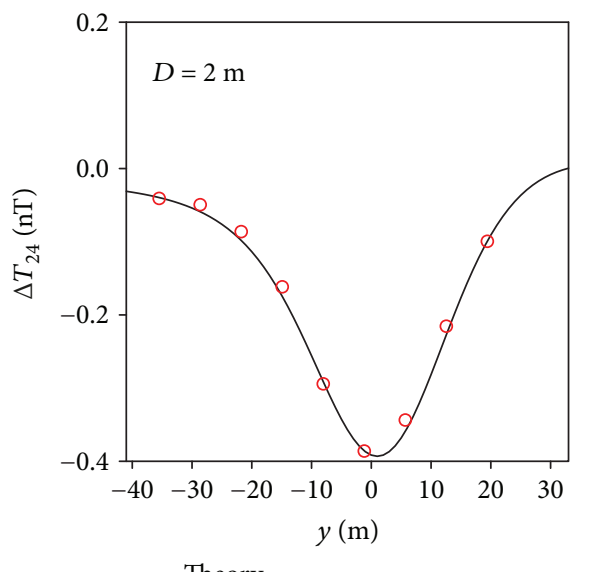

- Theory

- Experiment

(e) $D=2 \mathrm{~m}$, curves of $\Delta T_{24}$ and $y$

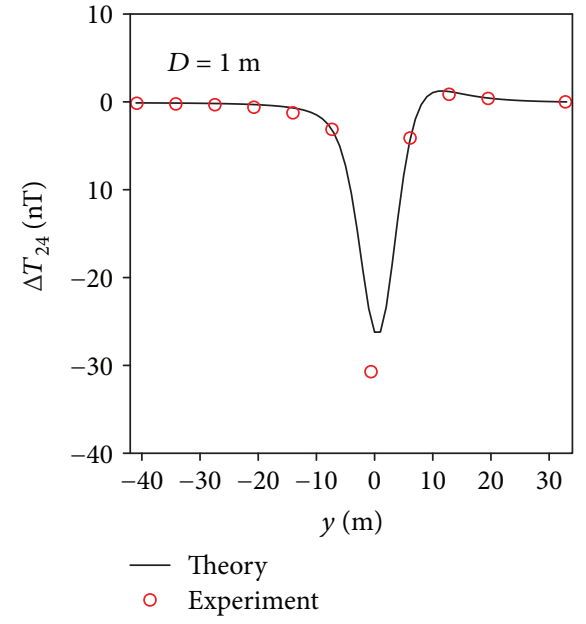

(b) $D=1 \mathrm{~m}$, curves of $\Delta T_{24}$ and $y$

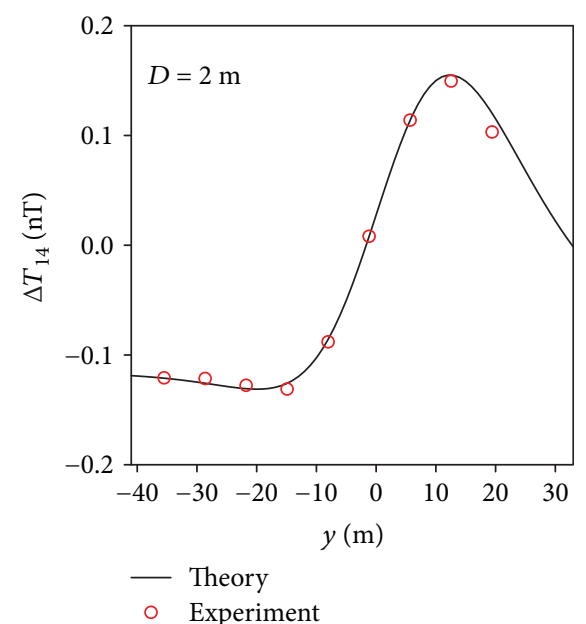

(d) $D=2 \mathrm{~m}$, curves of $\Delta T_{14}$ and $y$

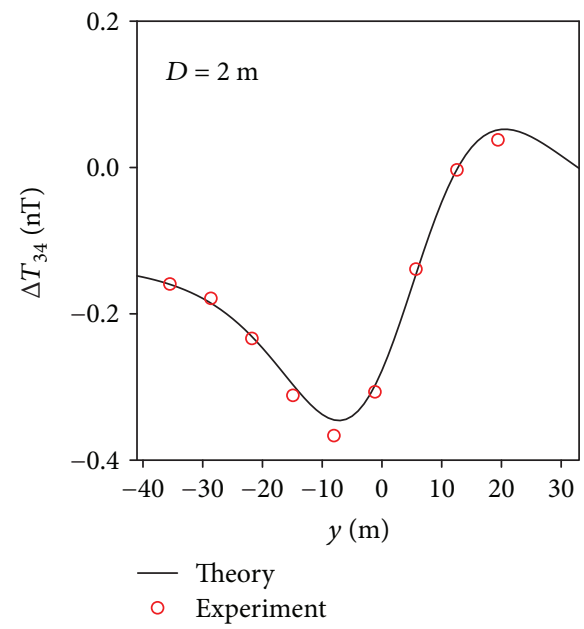

(f) $D=2 \mathrm{~m}$, curves of $\Delta T_{34}$ and $y$

Figure 8: Continued. 


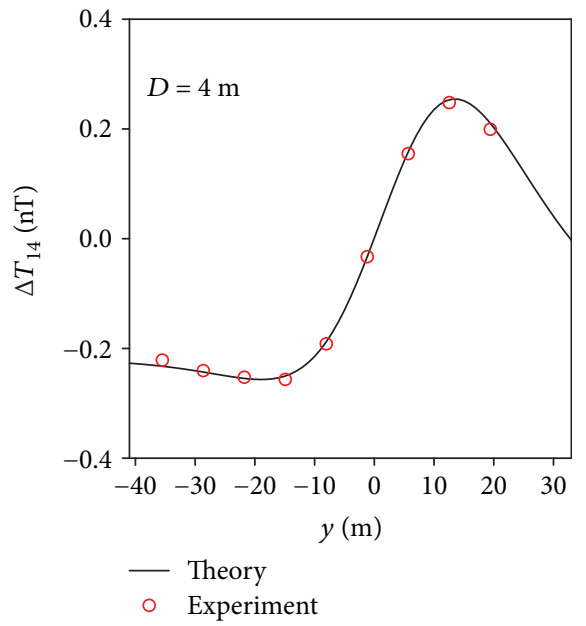

(g) $D=4 \mathrm{~m}$, curves of $\Delta T_{14}$ and $y$

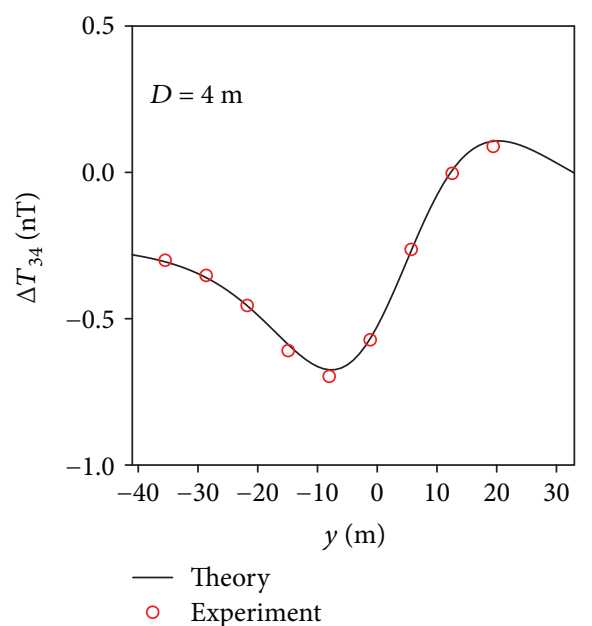

(i) $D=4 \mathrm{~m}$, curves of $\Delta T_{34}$ and $y$

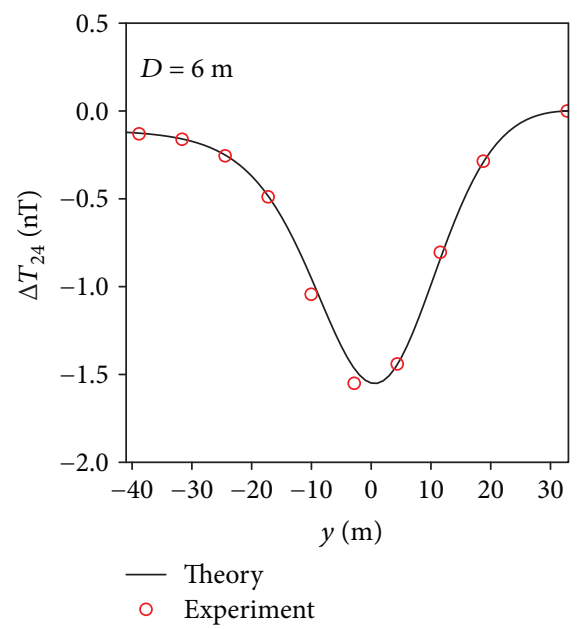

(k) $D=6 \mathrm{~m}$, curves of $\Delta T_{24}$ and $y$

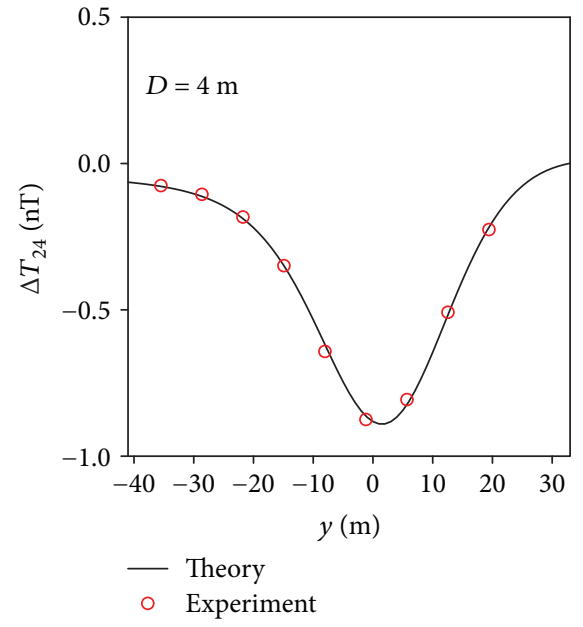

(h) $D=4 \mathrm{~m}$, curves of $\Delta T_{24}$ and $y$

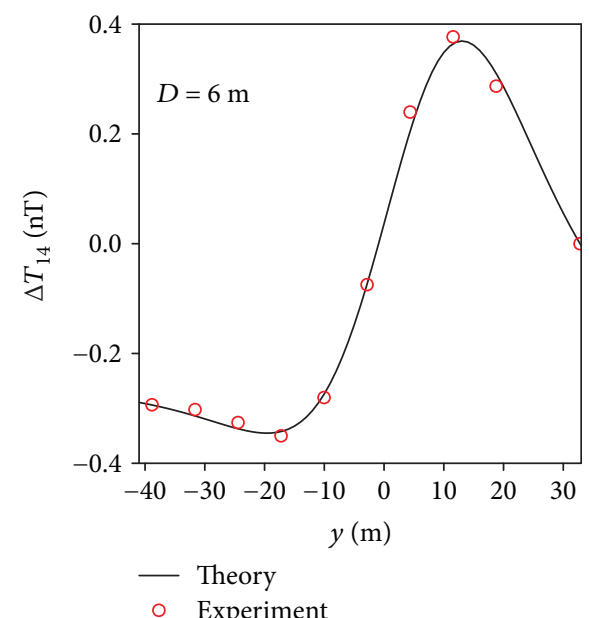

(j) $D=6 \mathrm{~m}$, curves of $\Delta T_{14}$ and $y$

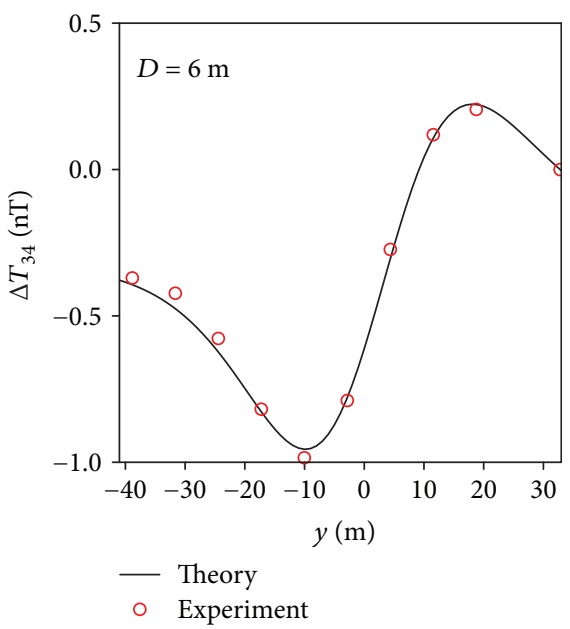

(l) $D=6 \mathrm{~m}$, curves of $\Delta T_{34}$ and $y$

Figure 8: Experimental data and theoretical curves of $\Delta T_{i j}$.

simulation with MathCAD and the target location $y$. Within the scope of the car moving, the curve of the simulation is consistent with the experimental data. It proves that the far-field model of magnetic dipole is correct in describing the magnetic field model of the car. In the experimental process, the time-varying and uneven spatial distribution of geomagnetic total field is eliminated, which proves that formula (5) and formula (6) are correct. 


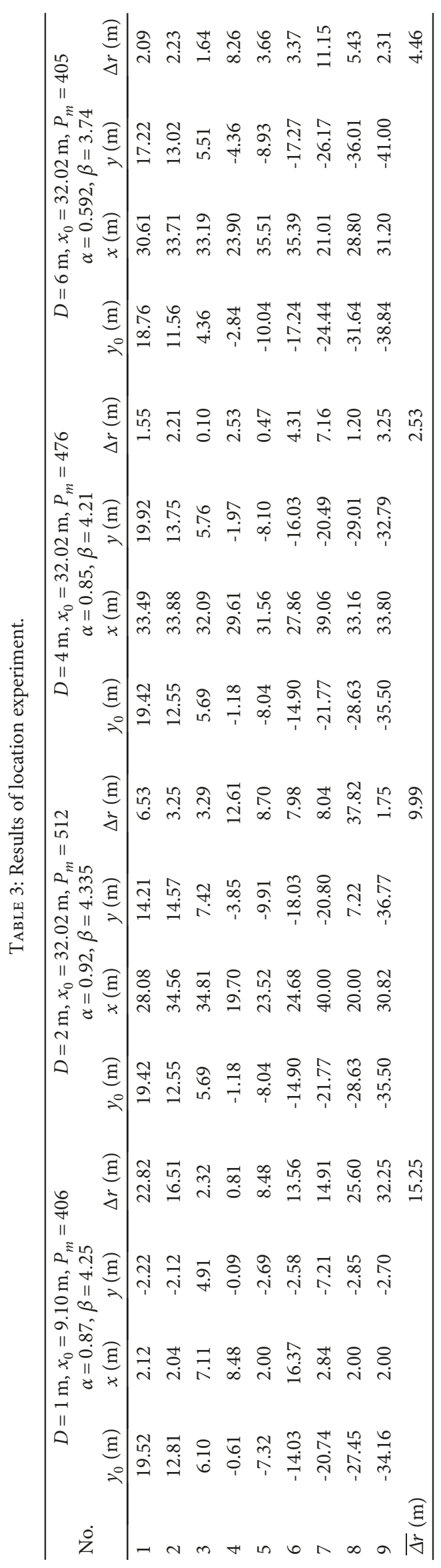




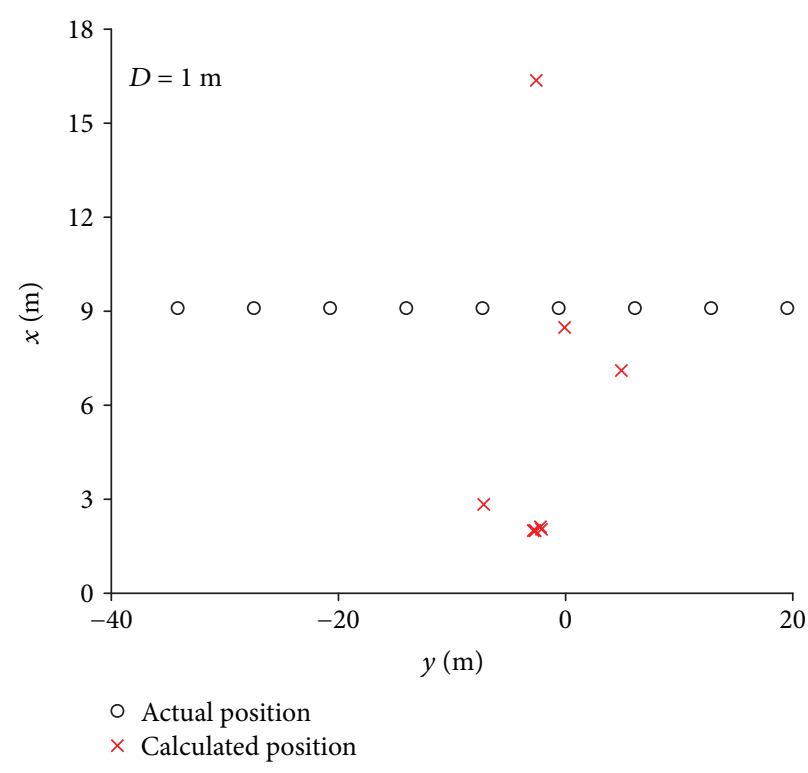

(a) When $D=1$, results of location experiment

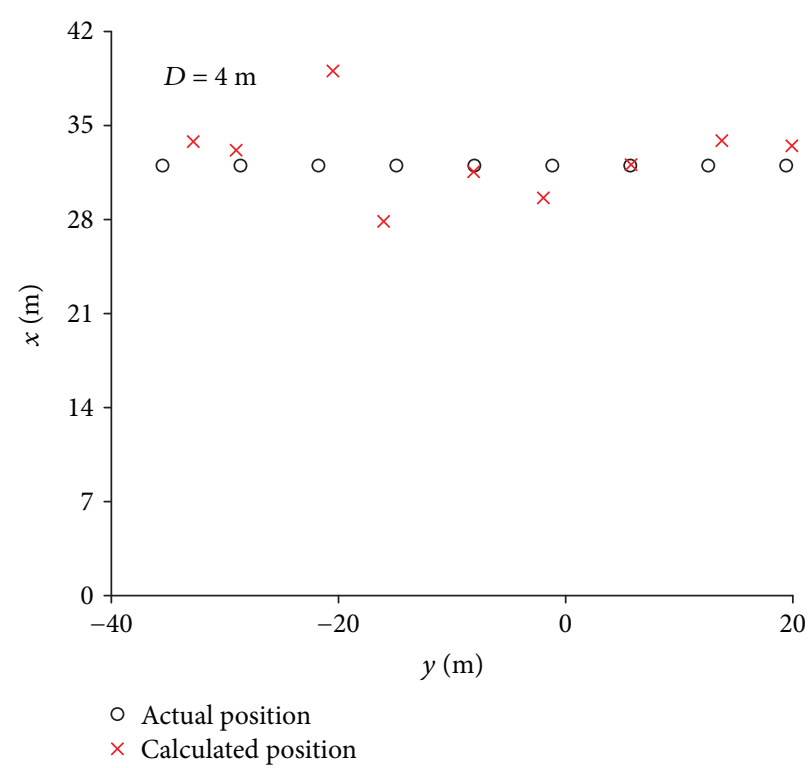

(c) When $D=4$, results of location experiment

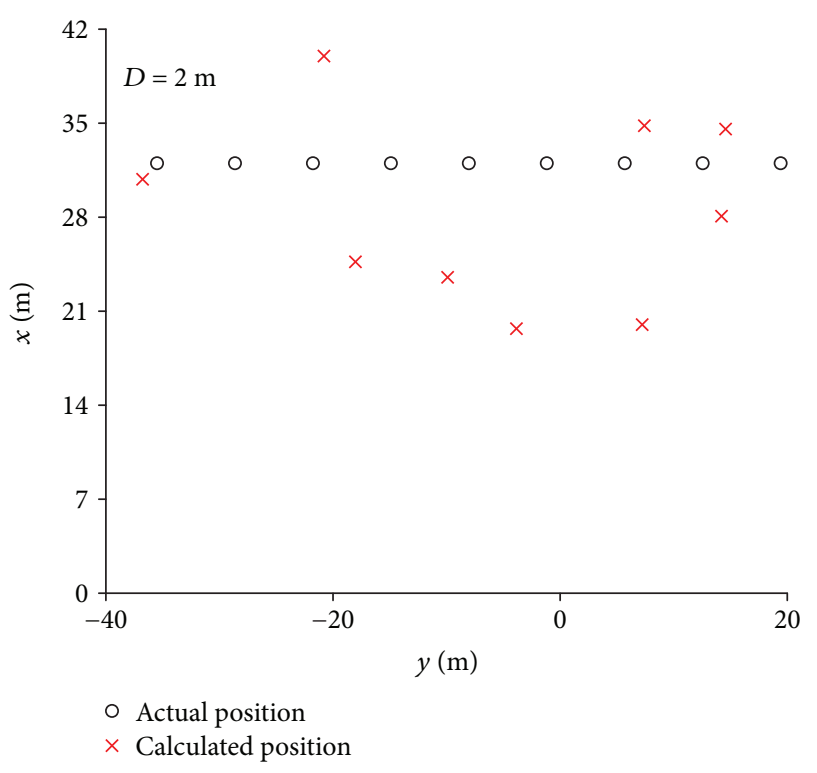

(b) When $D=2$, results of location experiment

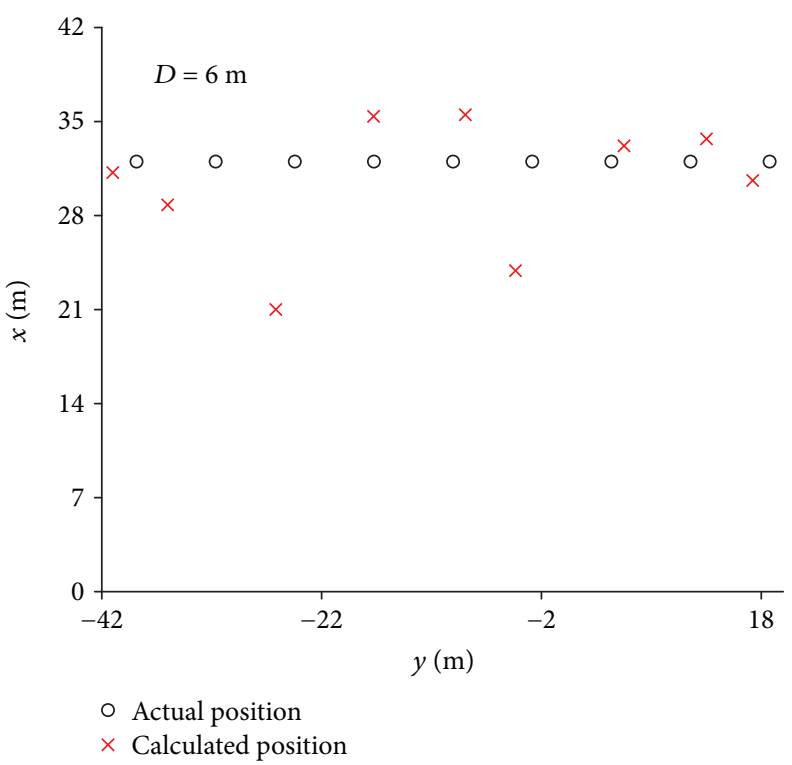

(d) When $D=6$, results of location experiment

Figure 9: Results of location experiment.

According to formulae (5) and (6), the position $(x, y)$ of the target at each time is calculated using the experiment data, as shown in Figure 8. Formula (6) is a high-order nonlinear equation, which is difficult to solve. The software LINGO is used for the numerical solution, and the location results based on the magnetic field are shown in Table 3.

In Table $3,\left(x_{0}, y_{0}\right)$ is the actual location of the target car, and $(x, y)$ is the target location calculated using the geomagnetic measurements, with the unit m. $\Delta r$ is the distance between the measuring point and the actual location of the target; $\overline{\Delta r}$ is the average of the distance, with the unit $\mathrm{m}$. The smaller the $\overline{\Delta r}$, the higher the positioning accuracy.
Based on the proposed method of determining the optimum aperture, the minimum array aperture $D_{\min }=4.5 \mathrm{~m}$ satisfied the requirement in the simulation when the location accuracy is $1 \mathrm{~m}$. In the table, it can be seen that $\overline{\Delta r}$ decreases from $15.25 \mathrm{~m}$ to $2.53 \mathrm{~m}$ when $D$ increases from $1 \mathrm{~m}$ to $4 \mathrm{~m}$. Then the location accuracy is getting higher, which meet with the simulation results. However, when $D$ increases to $6 \mathrm{~m}$, the location accuracy is not higher, and $\overline{\Delta r}=4.46 \mathrm{~m}$. It is because of the multivalueness of equation (5), and LINGO does not converge to the real optimal solution. In order to solve this problem, the additional conditions or criteria should be added to the algorithm. One way is to increase the number of linearly independent magnetic field sensor. 


\section{Conclusion}

The method of constructing an array of total field magnetometers to locate a target is proposed. This method is based on a magnetic dipole model of far field. We filtered out the time-varying and uneven spatial distribution by the conjugate gradient algorithm. Then the method of determining an array aperture is proposed. We simulate the location algorithm and the process of determining an array aperture. The location experiment is carried out in the suburb. We choose $1 \mathrm{~m}, 2 \mathrm{~m}, 4 \mathrm{~m}$, and $6 \mathrm{~m}$, respectively, as the aperture. The experimental data is consistent with the theoretical curve. In the location algorithm, interactive linear and general optimization solver LINGO is used to solve the nonlinear equations. The location accuracy of four apertures is calculated, respectively, which basically agrees with the simulation. In the experiment, the CS-L cesium optically pumped magnetometers are used. The CS-L cesium optically pumped magnetometers have a high resolution. Also, the measurement is not affected by the temperature. The attitude of the magnetometers is not rigidly calibrated. In the location experiment, the diagonal of the car is $4.64 \mathrm{~m}$ and the magnetic moment is about $405 \sim 512 \mathrm{Am}^{2}$. The vertical distance between the array and the car is $32.02 \mathrm{~m}$, and the parallel distance is $-41 \mathrm{~m}$ to $32.8 \mathrm{~m}$. The experimental location results are shown in Figure 9 . When the array aperture is $4 \mathrm{~m}$, the average deviation of the magnetic moment in locating the car is $2.53 \mathrm{~m}$. In a word, the method of locating the target and determining the aperture is feasible.

\section{Data Availability}

The data used to support the findings of this study are available from the corresponding author upon request.

\section{Conflicts of Interest}

The authors declare that they have no conflicts of interest.

\section{Supplementary Materials}

The measurement values of magnetometers in the location experiment. The data are the original experimental data which are measured by the CS-L array in Figure 2. Based on these data, we get Figures 3 and 8 . After processing the data, we get the results of location experiment in Figure 9. (Supplementary Materials)

\section{References}

[1] O. B. Novik, S. V. Ershov, M. N. Volgin, and A. O. Novik, "Magnetic location of a possible earthquake epicentre area: a mathematical model," Geomatics, Natural Hazards and Risk, vol. 7, no. 1, pp. 113-126, 2014.

[2] G. H. Wang, H. Zhu, and Z. D. Guo, "Analysis of magnetic dipole approximate range for a submarine," Journal of Naval University of Engineering, vol. 20, no. 5, pp. 60-63, 2008.

[3] X. T. Weng and M. F. Cao, "A resarch on the calculation of the induced magnetic field of a submarine," Journal of Shanghai Jiaotong University, vol. 28, no. 5, pp. 69-76, 1994.
[4] Z. N. Yao, D. M. Liu, D. S. Liu, and X. L. Zhu, "A real-time magnetic localization method of underwater non-cooperative magnetic targets based on unscented particle filter," Acta Physica Sinica, vol. 63, no. 22, pp. 309-314, 2014.

[5] Y. Sun, C. H. Xiao, and G. H. Zhou, "An active method and its analytical formulas of magnetic sensor positioning using the magnetic source," Hydrographic Surveying and Charting, vol. 32, no. 4, pp. 25-28, 2012.

[6] Y. Wu and W. Shi, "On calibration of three-axis magnetometer," IEEE Sensors Journal, vol. 15, no. 11, pp. 6424-6431, 2015.

[7] H. Pang, M. Pan, C. Wan, J. Chen, X. Zhu, and F. Luo, "Integrated compensation of magnetometer array magnetic distortion field and improvement of magnetic object localization," IEEE Transactions on Geoscience and Remote Sensing, vol. 52, no. 9, pp. 5670-5676, 2014.

[8] Z. Zalevsky, Y. Bregman, N. Salomonski, and H. Zafrir, "Resolution enhanced magnetic sensing system for wide coverage real time UXO detection," Journal of Applied Geophysics, vol. 84, no. 4, pp. 70-76, 2012.

[9] W.'a. Yang, C. Hu, M. Li, M. Q.-H. Meng, and S. Song, "A new tracking system for three magnetic objectives," IEEE Transactions on Magnetics, vol. 46, no. 12, pp. 4023-4029, 2010.

[10] G. Iacca, F. L. Bakker, and H. Wörtche, "Real-time magnetic dipole detection with single particle optimization," Applied Soft Computing, vol. 23, no. 5, pp. 460-473, 2014.

[11] M. Beiki, D. A. Clark, J. R. Austin, and C. A. Foss, "Estimating source location using normalized magnetic source strength calculated from magnetic gradient tensor data," Geophysics, vol. 77, no. 6, pp. J23-J37, 2012.

[12] N. Wahlstrom and F. Gustafsson, "Magnetometer modeling and validation for tracking metallic targets," IEEE Transactions on Signal Processing, vol. 62, no. 3, pp. 545-556, 2014.

[13] A. M. Zhang, W. T. Chen, W. Q. Yu, and C. B. Guo, "The equivalent calculation for the induced magnetic fields of a ship hull module," Chinese Journal of Ship Research, vol. 9, no. 3, pp. 109-112, 2014. 


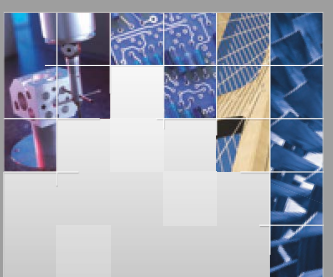

\section{Enfincering}
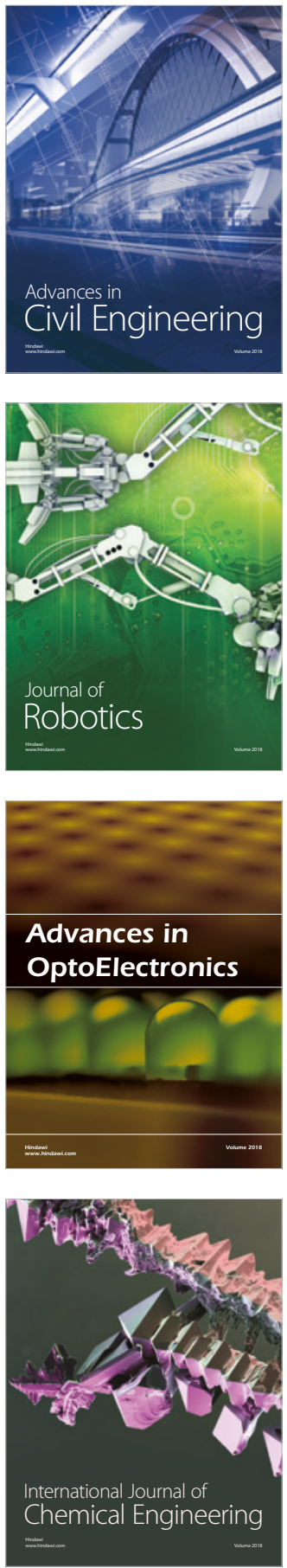

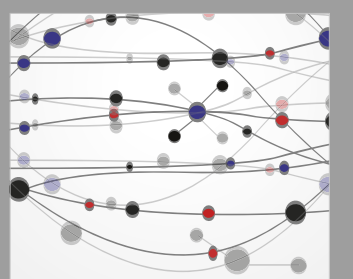

\section{Rotating \\ Machinery}

The Scientific World Journal

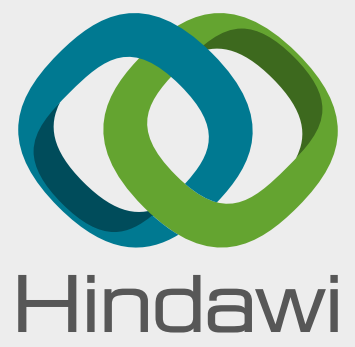

Submit your manuscripts at

www.hindawi.com
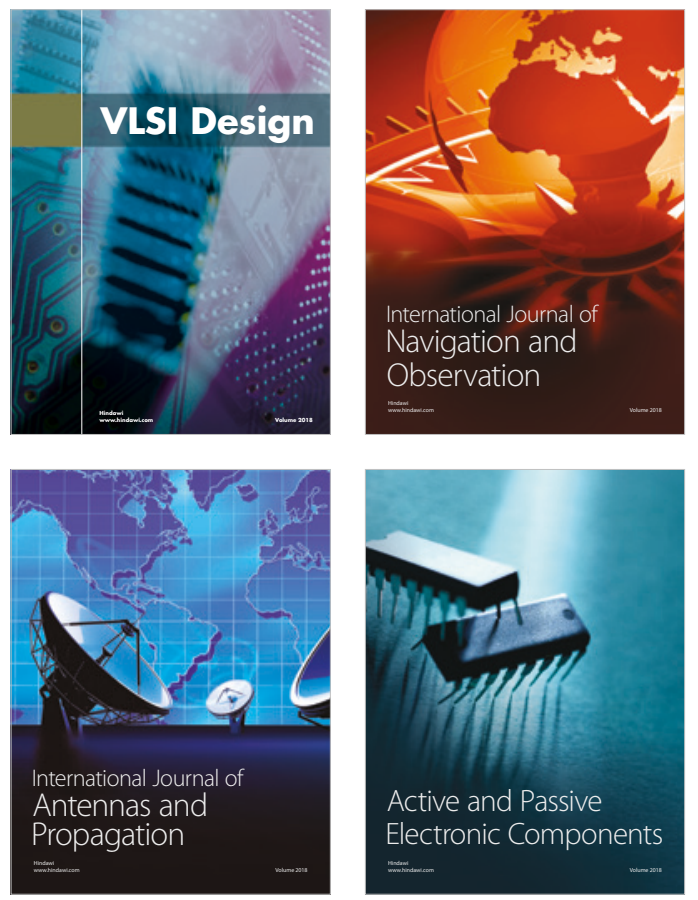
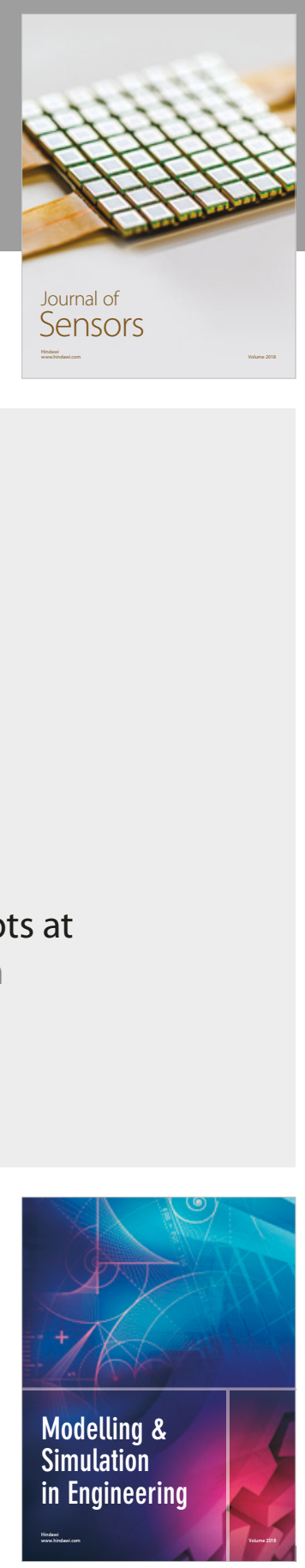

\section{Advances \\ Multimedia}
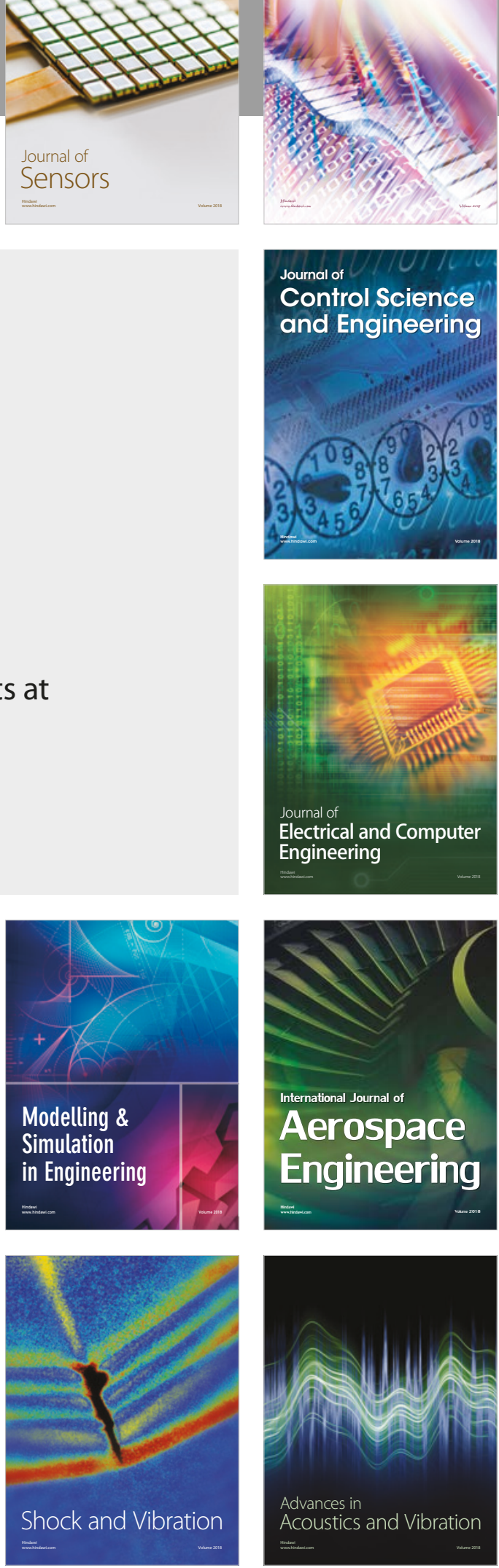